\title{
Recruitment of Minority Women and Their Main Sexual Partners in an HIV/STI Prevention Trial
}

\author{
SUSAN S. WITTE, Ph.D., ${ }^{1}$ NABILA EL-BASSEL, D.S.W. ${ }^{1}$ LOUISA GILBERT, M.S.W. ${ }^{1}$ \\ ELWIN WU, Ph.D., ${ }^{1}$ MINGWAY CHANG, M.A., ${ }^{1}$ and PETER STEINGLASS, M.D. ${ }^{2}$
}

\begin{abstract}
Recruiting heterosexual couples into randomized clinical trials (RCTs) to test the efficacy of $\mathrm{HIV} /$ sexually transmitted infection (STI) prevention interventions is a challenge that requires innovative strategies and consideration of ethical issues, including participant safety and confidentiality.

Methods: This paper provides a brief review of the literature on minority and couple RCT recruitment and describes the development (preparation phase and protocol development) and implementation (strategies employed and barriers) of a recruitment protocol that safely enrolled 217 predominantly African American and Latino heterosexual couples into a relationship-based, HIV/STI prevention study.

Results: The success of this recruitment protocol with no reported adverse events demonstrates the feasibility of engaging urban minority women and men in RCTs. This study builds on a small literature base articulating specific couple recruitment strategies.

Conclusion: More research delineating and testing specific strategies for recruiting defined populations into clinical trials is needed to advance the science of study recruitment and improve generalizability of research findings.
\end{abstract}

\section{INTRODUCTION}

$\mathbf{T}$ HERE IS INCREASING INTEREST in the development and evaluation of couple-oriented interventions in the field of HIV prevention. ${ }^{1-11}$ As innovations in HIV prevention intervention protocols and targets of change evolve, there is a need to examine and understand the attendant adaptations to recruitment protocols and strate- gies needed to run rigorous efficacy trials. Concurrently, there is growing emphasis on ensuring that women, racial/ethnic minorities, and other socioeconomically disadvantaged groups are represented in clinical research. ${ }^{12,13}$ Recruiting minority couples into HIV-related randomized clinical trials (RCT) to test the efficacy of coupleoriented prevention interventions presents numerous challenges. More research aimed at de-

\footnotetext{
${ }^{1}$ Social Intervention Group, Columbia University School of Social Work, New York, New York.

${ }^{2}$ Ackerman Institute for the Family, New York, New York.

This study was supported by grant MH57145 from NIMH awarded to N.E.-B.
} 
lineating and testing specific strategies for recruiting such defined populations into clinical trials is needed. ${ }^{14}$ This paper bridges an existing gap to such future scientific endeavors by describing the development of a couple RCT recruitment protocol. Specifically, this paper (1) provides a brief review of the extant literature on minority and couple recruitment in RCTs, (2) describes a recruitment protocol to safely enroll 217 predominantly African American and Latino heterosexual couples into Project Connect, a relationship-based $\mathrm{HIV}$ /sexually transmitted infections (STI) prevention intervention study designed for women and their regular sexual partners, and (3) provides implications of the findings for the improvement of couple recruitment strategies in future HIV prevention intervention trials.

Barriers to the recruitment of minority participants into RCTs in the United States are highlighted by the historical and contextual experiences of minority groups, particularly of African Americans. These include (1) individual barriers, such as feeling that procedures are too invasive or feeling fearful about research, ${ }^{14-16}$ (2) research barriers, such as knowledge of the abuses noted in research historically, including the Tuskegee Syphilis Experiment, and generally negative attitudes toward research and researchers, ${ }^{14-19}$ (3) sociocultural barriers, such as perceived racial and ethnic discrimination, and fear and mistrust of the medical system or other systems where research is conducted, ${ }^{14-16,19}$ and (4) economic barriers, such as transportation costs or lack of access to healthcare in general. ${ }^{14,16}$ These barriers appear to be borne out based on findings from a recent study that directly asked participants about their attitudes toward clinical trials ${ }^{20}$ and may underlie the significantly lower rates of participation among African Americans and Latinos in HIV clinical trials. ${ }^{21}$

Although numerous barriers have been documented, so too have recommended recruitment strategies. A synthesis of minority RCT recruitment strategies representing over 38 RCTs implemented between 1987 and 1994 highlights four strategic targets: individual, researcher, study site, and community. ${ }^{14}$ Individual strategies include incorporating an understanding of cultural beliefs, practices, and lifestyle into promotional materials; offering compensation; and providing child care and transportation. Researcher strategies include demonstrating sensitivity to partici- pants' safety concerns, usefulness of the project, and using ethnically and racially matched recruitment staff. Study site strategies include involving site staff in the design and procedure development for the trial and clearly defining the site staff role. Community strategies include involving community members and organizations in recruitment procedures and protocols and demonstrating a benefit to the community from the trial. ${ }^{14}$ These strategies have been applied in research among minority individuals ${ }^{15,19,22,23}$ and families ${ }^{16,23}$ and offer a beginning literature base on recruitment protocols targeting minority research participants. ${ }^{16,24}$ It is critical to build on this base by testing recruitment protocols for RCTs and discussing and demonstrating their relative feasibility and success in achieving sample sizes in particular to address the gap in the recruitment of minority couples.

Although most literature on minority RCT recruitment describes strategies for enrolling African American participants, the literature on recruitment of Latino RCT participants is growing. Cultural factors that present potential barriers to recruitment within the Latino community include the importance of strong and traditional family values (familismo), ${ }^{25,26}$ respect toward male figures (personalismo), particularly the role of the father (machismo), in family decision making, ${ }^{25-27}$ and the need for Spanish-speaking research staff and Spanish language research materials. ${ }^{25}$ Issues of familismo, personalismo, and machismo in family decision making are potential barriers to couple recruitment, as they suggest the need for the assent (or consent) of male heads of household, who would then recruit additional family members. When the emphasis of couple studies is on shared or joint decision making, such recruitment strategies may contradict the intention of the research process or goals. Overcoming such cultural barriers requires innovative strategies, best drawn from within the community, ${ }^{28,29}$ that take these factors into consideration. ${ }^{25-27}$ Currently, no reports that detail and describe the success of recruitment strategies to target Latino couples in RCTs, specifically those testing HIV / AIDS interventions, are available.

Literature on couple RCT recruitment generally comes from two areas: marital and family therapy interventions ${ }^{30-32}$ and reproductive health interventions. ${ }^{1,9,17,33,34}$ Few studies from marital therapy provide detailed descriptions of couple recruitment strategies. ${ }^{31,32}$ Moreover, 
these studies relied explicitly on married couples, targeting Caucasian samples, thus leaving a gap in the knowledge base for African American and Latino communities as well as nonmarried, longer-term partners.

Preloran et al. ${ }^{29}$ provide the first published description of successful couple recruitment strategies we identified. They were used to enroll Latino couples into a qualitative study of amniocentesis decision making among Mexican immigrant couples. Drawing on male and female cultural scripts, four distinct strategies are described: (1) a standard approach, where one partner is contacted in person and the researcher follows up with both partners by phone, (2) an on the spot approach, where both partners are present at the clinic recruitment setting and can be recruited together, (3) a corecruitment strategy, where a woman is first recruited in person, then she will broach the issue with her partner, and the researcher completes the process, and (4) a brokering strategy, where a woman is first recruited in person, and then she independently recruits her male partner without additional support from the research team. ${ }^{29}$

Recent work by McMahon et al. ${ }^{35}$ provides the first published description of the recruitment of urban street-based drug-using couples into public health programming. This work took place between 2001 and 2003, and a main concern addressed in this work is the use of couple verification screening (CVS) to verify the legitimacy of the dyadic relationship.

These articulated couple recruitment strategies are similar to those devised and implemented in Project Connect between 1997 and 2001, as described later. These similarities give weight to their utility for minority couple recruitment in both qualitative and quantitative studies, including RCTs, and provide a base on which to build models for testing the effectiveness of such articulated recruitment protocols.

Unique to the Project Connect recruitment protocol is attention to concerns related to HIV/STI prevention intervention, which may be more sensitive and difficult to negotiate with couple participants than amniocentesis decision making or more general public health programming. These circumstances require consideration of genderbased issues of power, control, and dominance in sexual relationships. HIV/STI risk reduction interventions address sensitive topics, including condom use, that are often emotionally charged, raising concerns of infidelity, lack of trust, or other issues that threaten relationship stability. ${ }^{36,37}$ Ignoring these issues in the recruitment process could cause conflict between couples and subsequent danger related to potential intimate partner violence (IPV). There is a growing body of literature underscoring the relationship between IPV and HIV/STI risk behavior. ${ }^{38,39}$ Thus, although recruitment of male partners is critical to sample size achievement, more compelling grounds for defining and monitoring a formal recruitment protocol are to ensure participant safety. With concern for gender-based issues of power, control, and dominance, the original study design described in this paper called for recruitment of women first and then subsequent male partner recruitment. This would put control of the recruitment process more in the hands of the woman, thereby hopefully minimizing any potential coercion from male partner to female partner to participate.

A second ethical issue raised by recruiting one partner first involves respecting the confidentiality of the partner to be enrolled. Compliance with federal human subjects regulations requires special attention to the procurement of contact information on all human subjects researchers intend to engage in a research study. ${ }^{13}$ Careful definition of strategies for partner engagement with respect to human subjects issues is crucial.

\section{MATERIALS AND METHODS}

Taking into account the extant literature on minority and couple recruitment, as well as ethical issues, including gender-based issues of power, control, and dominance in relationships, we devised and employed a recruitment protocol development phase to ensure the cultural relevance, utility, and safety of the couple recruitment process on three levels: (1) research staff, (2) study site staff, and (3) participant couples. In this third level, we combine the goals of both individual and community level strategies as articulated by Swanson and Ward. ${ }^{14}$ Beyond existing literature, data used to develop this recruitment protocol come from predominantly qualitative (pilot study focus groups) and descriptive (debriefing with research staff and pilot study participants) sources.

In the process of preparing research staff, first, we selected the most experienced members of our 
recruitment staff to conduct project enrollment. Staff roles were developed to be full-time, allowing staff to become well known and a constant presence within the community. Staff participated in over 20 hours of training using the latest recruitment technology for HIV prevention trials. ${ }^{40}$ Skill-building approaches, including modeling best practice recruitment techniques, and role-playing recruitment with a variety of participant types was implemented. Recruiters were African American or Latino and familiar with the community in which recruitment took place. The training emphasized the important role the recruiter plays in not only the scientific integrity of the study (rigorous recruitment strengthens external validity) but also as the face of the study and the importance of the relationship between study staff and participants. Language, body language, dress, interaction techniques to demonstrate respect for and to allow the self-determination of each participant in the research process were emphasized, as was the overall goal of the project: to improve health and relationships by preventing STIs, including HIV. During the trial, weekly research team support meetings provided ongoing encouragement, assured oversight and accountability of recruitment goal achievement, and provided troubleshooting for the most effective interactions with potential study participants.

Site staff preparation was facilitated by the advantage of choosing for this RCT an outpatient clinic where our research team had operated for a number of years, thus making them familiar with clinic operations and staff. Although not all studies have this advantage, a key to recruitment in host settings is familiarity of study staff with site staff and having study staff that are comfortable and familiar in the recruitment setting. Research staff hosted a series of luncheon meetings where the study was presented and discussed as forums to engage site staff in the process of defining collaborative recruitment efforts and to encourage them to buy in to the importance of the study. During these meetings, study staff clarified that the recruitment process would require no additional responsibilities for site staff. Study staff highlighted to site staff the benefits of study participation for site clientele, also clarifying the human subjects review process for the safety and confidential protection of clients/participants. This process successfully engaged site staff as partners in the recruitment effort.
Participant preparation began with a qualitative pilot study conducted during the first year of this RCT with several goals: (1) to inform the specific language, concepts, and skills for the intervention, (2) to attend to the cultural relevance of intervention content, and (3) to articulate recruitment strategies sensitive to issues of gender, power, and culture and to explore and address potential recruitment barriers. Results of the first two goals are described elsewhere (see refs. 5 and 28 for more information on intervention content). Results on the development of couple recruitment strategies are reported here. The pilot study involved 16 women and 13 of their male partners (55\% African American and 35\% Latino) who were asked to serve as study consultants by attending a series of three focus groups. Focus groups were a combination of single-gender and mixed-gender groups to allow safe exploration of the gender dynamics of sensitive topics or concerns, such as partner recruitment barriers or discussion of HIV/STI risk behaviors. Participants were asked what specific strategies could be used by women to recruit and engage their male partners in an HIV/STI prevention study and what constituted likely recruitment barriers. The preliminary protocol implemented to recruit the participants for this pilot study was refined with study feedback and became the main phase recruitment protocol described.

Pilot results found that the most commonly mentioned motivators for participation by both women and men were appeals to social conscience, financial compensation, and an opportunity to improve their relationship. These findings informed motivational statements used to engage eligible women and their partners. Pilot results also defined terminology used for screening and recruitment protocols (e.g., main partner), actual recruitment strategies, and tools used in the recruitment process (e.g., fliers, male partner recruitment letter).

The final main phase recruitment protocol involved three steps: (1) screening for the woman's eligibility, (2) recruitment of her male partner, and (3) completion of a baseline assessment interview with both partners.

Identifying women as the initial targets of recruitment was based on the original design of the study (taking into consideration gender-based issues of power, control, and dominance in relationships), which had been approved by funding source officials and by the institutional review 
boards at both research and study site and was reinforced as the preferred strategy by couples consulted in the pilot phase. Screening for this study took place in an outpatient clinic building at a large, New York City hospital. All female patients entering the outpatient building were approached by project staff, handed a project flier, and invited to complete a brief screening questionnaire. Site staff assisted in this process by asking clients if they were interested in hearing more about the project upon completing their clinic appointments. The project was introduced as "a study to learn more about relationships between women and men in the community, particularly the ways to help long-term couples maintain safe and healthy relationships." Approximately one of three women approached indicated interest. These women were asked to come to a private office where they completed a 10-minute, face-to-face screening administered by a female interviewer. Participants were reimbursed with the cash equivalent of a roundtrip subway fare.

A woman was eligible for the study if she (1) was between the ages of 18 and 55 years old, (2) had a main regular sexual partner (i.e., a spouse, lover, or boyfriend) with whom she had been involved for at least 6 months prior to screening and with whom she intended to stay for at least 1 year following screening, (3) had had sexual intercourse with that partner during the 6 months prior to screening, (4) had not used condoms on all of these occasions during the 6 months prior to screening, (5) was a patient at one of the outpatient clinics, and (6) had not experienced severe physical or life-threatening abuse from that partner within the 6 months prior to screening, assessed using the Revised Conflict Tactics Scales. ${ }^{41}$ (Women who reported severe abuse were excluded from the study. Their participation in the intervention might have increased the risk of injury. Involvement in couples-based interventions is contraindicated for women experiencing severe abuse. ${ }^{42,43}$ Women who reported mild or moderate abuse were carefully counseled and monitored by recruitment staff who explored the likelihood of study recruitment exacerbating or initiating violence within the dyad.)

A final eligibility criterion required that a woman reported knowing or worrying that her main partner (1) had sex with someone else in the 90 days prior to screening, (2) had had an STI, such as gonorrhea, syphilis, or chlamydia during the
90 days prior to screening, (3) had injected drugs during the 90 days prior to screening, and/or (4) was HIV-infected. By using these eligibility criteria, we aimed to recruit a sample of women who reported behavior consistent with high risk for STIs or sexual HIV transmission to or from her main partner.

Eligible women received a detailed explanation of study participation requirements: recruit her partner, complete a baseline assessment interview, be randomized to one of three study conditions, participate in intervention sessions, and complete two follow-up assessment interviews at 3 and 12 months postintervention. The three study conditions included (1) couple sessions, where both the woman and her partner received the intervention delivered by a female facilitator, (2) woman-alone sessions, where the woman without her sexual partner received the same intervention delivered by a female facilitator, or (3) an educational session, where the woman, without her sexual partner, received one HIV/AIDS information session. Participants were given the opportunity to ask questions, and staff explored and problem-solved potential barriers to participation. Each eligible woman also completed a detailed contact sheet on both herself and the main partner to whom she referred during the screening assessment. This contact sheet included specification of contact parameters for protection of confidentiality: for example, "May we send mail to this address?" "May we call you at this phone number?" "Who shall we say is calling so you will know it is from the project?" These types of parameters are particularly important when dealing with studies related to HIV/STIs-first, because of the perceived and actual stigma associated with issues related to HIV/STI within many communities, ${ }^{44}$ and second, in order to demonstrate how seriously research staff take participant confidentiality and autonomy.

Eligible, interested women were asked if they believed that their main partner would be interested in participating and then were offered several strategies for partner recruitment (Table 1). The first male partner recruitment approach, a brokering strategy, named by Preloran et al., ${ }^{29}$ was for each woman to describe the project to her partner and to engage his participation on her own. To assist in this process, some women chose a brokering plus invitation approach, where the woman hand-delivered a formal study invitation letter on institutional letterhead, addressed to her 
Table 1. Male Partner Recruitment Strategies

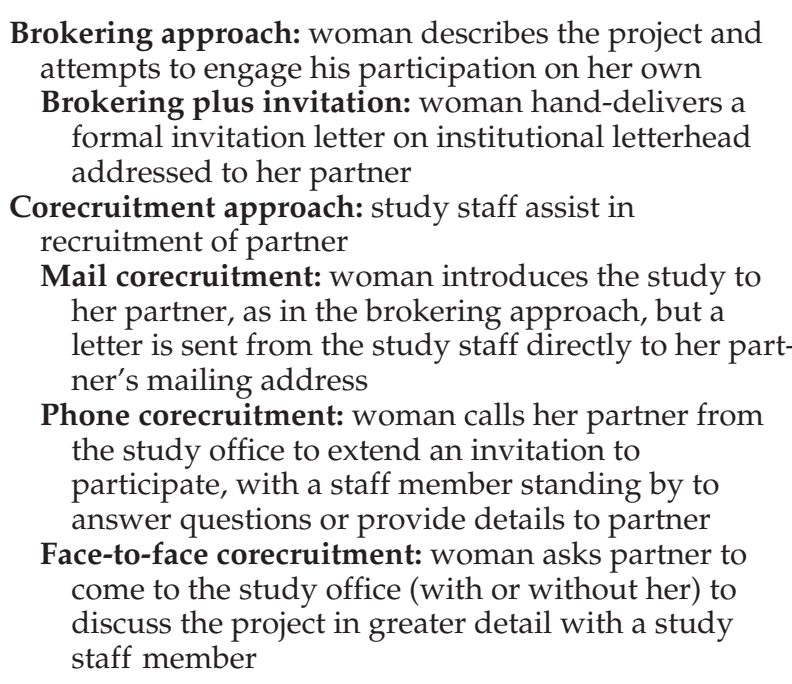

partner. The letter introduced the study, described its purpose, and provided a telephone number to call for more information if desired. The letter requested that he call the project as soon as possible to verify the couple's participation or to clarify any questions. Couple consultants provided the language for this letter during the pilot phase.

The next three strategies described are similar to the corecruitment strategy employed by Preloran et al. ${ }^{29}$ However, we break corecruitment down into mail, phone, or face-to-face strategies. Mail corecruitment involved having the woman introduce the study to her partner, as in the brokering approach, but to have the invitation letter sent directly to her partner's mailing address from the study staff. Phone corecruitment involved having the woman call her partner from the study office to extend an invitation to participate, with either a male or female staff member standing by to answer any questions or to provide details about the study to her partner (choice of gender of staff member determined by the woman). The face-to-face corecruitment strategy involved having the woman ask her partner to come to the study office (with or without her) to discuss the project with a study staff member in more detail.

Women anticipating difficulty recruiting their partner were supported in a trouble shooting session by male study staff. Male staff role-played with women each of the strategies that she could use to motivate partner participation, with em- phasis on motivational statements highlighting potential benefits, such as the altruistic role of participation, financial compensation, and the potential to strengthen their relationship.

If neither the woman nor her partner called to set up a baseline assessment appointment, staff contacted the woman by telephone and by mail within the week to offer assistance with partner recruitment or to confirm an assessment interview date. Male and female partners were interviewed simultaneously, but separately, in private rooms with same-gender interviewers. Prior to the baseline interview, all participants signed a consent form that detailed confidentiality procedures and their rights as research participants, including receipt of financial incentives: $\$ 25 \mathrm{com}-$ pensation each for completing baseline interviews, \$20 each for each intervention session attended, and $\$ 40$ for each of two follow-up interviews completed (both men and women at 3 months, women only at 12 months). The recruitment process was considered complete when both the woman and her partner completed the baseline assessment and the couple was randomized to a study condition.

\section{RESULTS}

Between 1998 and 2000, a total of 2416 women were screened for the study, of which 388 (16\%) were eligible. Over half of eligible women $(n=$ $217,56 \%$ ) effectively engaged their male partner's participation, completed baseline assessments, and were randomized to a study condition. Sociodemographic characteristics of study participants are provided in Table 2. These eligibility and enrollment rates are consistent with prevention trials targeting populations at high risk of sexual HIV transmission. ${ }^{45}$ The three most frequently self-reported reasons for nonparticipation of eligible couples were (1) schedule conflicts, or an inability to attend the first two scheduled baseline appointment dates $(n=52$, $30 \%)$, (2) woman willing but partner unwilling to participate $(n=52,30 \%)$, and (3) woman no longer interested ( $n=50,29 \%)$ (Fig. 1). No adverse events related to study screening and enrollment were reported.

In a post hoc analysis, we took the quantitative measure of time elapsed between screening and randomization and categorized successfully recruited couples by the length of time from screen- 
Table 2. Sociodemographic Characteristics of Participants Randomized in the Study

\begin{tabular}{|c|c|c|c|c|c|c|}
\hline \multirow[b]{2}{*}{ Study arm } & \multicolumn{3}{|c|}{ Female } & \multicolumn{3}{|c|}{ Male } \\
\hline & Couples & $\begin{array}{l}\text { Women } \\
\text { alone }\end{array}$ & Education & Couples & $\begin{array}{l}\text { Women } \\
\text { alone }\end{array}$ & Education \\
\hline $\begin{array}{l}\text { Sample size } \\
\qquad(n=217 \text { couples })\end{array}$ & 81 & 73 & 63 & 81 & 73 & 63 \\
\hline Sociodemographics & \multicolumn{6}{|c|}{ Percentage } \\
\hline$<25$ years of age & 9.9 & 8.2 & 9.5 & 7.4 & 9.6 & 7.9 \\
\hline African American & 54.3 & 54.8 & 54.0 & 48.1 & 61.6 & 55.6 \\
\hline Hispanic & 38.3 & 43.8 & 36.5 & 42.0 & 31.5 & 39.7 \\
\hline$\geq$ High School or GED & 42.0 & 37.0 & 55.6 & $44.4^{\mathrm{a}}$ & $61.1^{\mathrm{a}}$ & $54.0^{\mathrm{a}}$ \\
\hline Never married & 67.9 & 57.5 & 52.4 & 56.8 & 54.8 & 54.0 \\
\hline Employed & 11.1 & 15.1 & 17.5 & $24.7^{*}$ & $45.2^{*}$ & $34.9^{*}$ \\
\hline Income $<\$ 5,000 /$ year & 64.2 & 72.6 & 68.3 & $56.3^{\mathrm{b}}$ & $46.6^{\mathrm{b}}$ & $47.6^{\mathrm{b}}$ \\
\hline
\end{tabular}

aSample sizes are 81, 72, and 63 for couples, women alone, and education, respectively.

*Significant difference between conditions at the $p<0.05$ level, with a chi-square test of association.

bSample sizes are 80, 73, and 63 for couples, women alone, and education, respectively.

ing to enrollment: (1) same day, (2) within 2 weeks, (3) 2-4 weeks, and (4) over a month. (A chi-square test comparing race/ethnicity [African American vs. Latino] with these four time categories indicates that there were no significant differences in time to enroll by ethnicity.) Revisiting qualitative notes kept on the contacts made with each couple during the recruitment process, we are able to provide some description of couples falling into each category.

Same day couples $(n=27,12 \%)$, like "on the spot" couples described by Preloran et al., ${ }^{29}$ were those where the partner either happened to have accompanied the woman to her clinic appointment or who lived within walking distance from the study site. In these situations, eligible women were able to bring their partners to the study office almost immediately for face-to-face corecruitment. Informing both partners about the study at the same time set an egalitarian tone for participation.

Most successfully recruited couples completed baseline assessment and randomization within 2 weeks of screening $(n=153,71 \%)$. Same day couples and those recruited within 2 weeks shared characteristics consistent with the participation motivators articulated by consulting couples: one or both partners were (1) motivated to serve the community, (2) interested in the financial compensation, (3) interested in strengthening their relationship, or (4) justly concerned about their HIV/STI risk potential. In some of these situa- tions, although the woman was enthusiastic, she anticipated that her partner might be reluctant. According to the protocol described, in these cases, a male staff member would first role-play with the woman to help prepare her for any negative comebacks on the part of her partner. In addition, the male staff person would offer to contact the male partner or to be available to speak with him in the event he had any questions.

More difficult recruitment situations involved couples enrolled 2-4 weeks postscreening $(n=$ $26,12 \%)$. Characteristics of these cases were that the woman was clearly concerned about her partner's willingness to participate, she indicated that she had no phone or mailing address, she was actively using drugs or alcohol, she had concerns about her immigration status becoming known, or one or both partners had too many other competing priorities to deal with in their current life context.

The fewest number of cases were those couples enrolled 1 month or more after screening $(n=11$, $5 \%)$ and required rescreening. These cases were similar to the 2-4 week cases, but also included those where a woman or her partner went to jail or moved, was hospitalized or entered drug or alcohol detoxification within the first or second week after screening, and had to be rescreened after being released several months later.

The research staff engaged several progressive strategies to engage difficult recruitment cases (Table 3). First, efforts to accommodate couples 


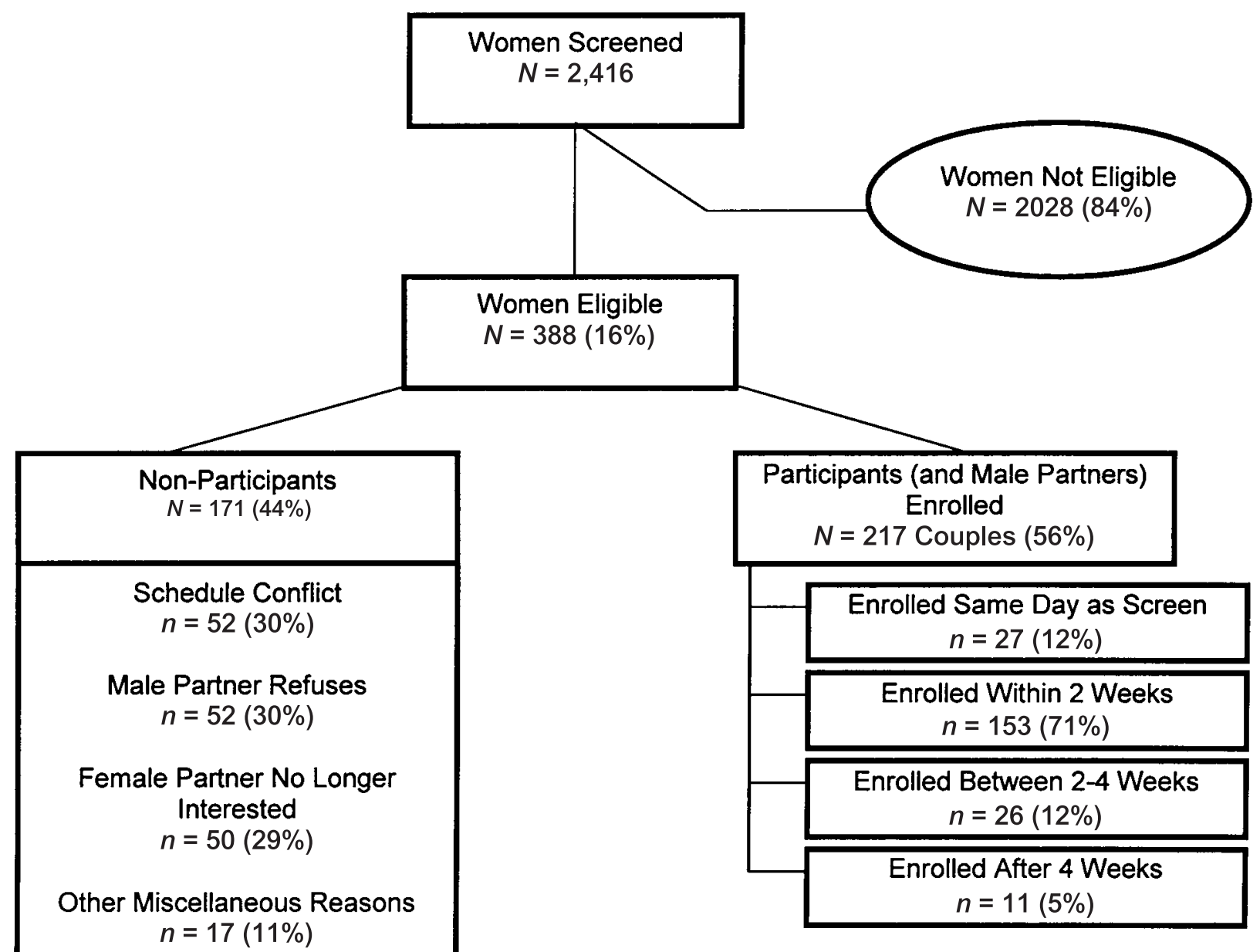

FIG. 1. Recruitment/enrollment process and outcomes.

whose scheduling was difficult included evening and weekend scheduling and time periods for drop-in baseline appointment times. Second, reluctant male partners or couples who lost interest postscreening were reminded of (1) the altruism of their participation, (2) the financial incentives provided, (3) the potential benefits for their relationship, and (4) the confidential nature of study participation. Confidentiality was emphasized for couples concerned about their immigration status coming to the attention of federal authorities. Third, a marketing approach that involved making multiple reminder telephone calls and mailings to communicate our continuing interest in couple enrollment was used. If all of the above failed, final special efforts were engaged including (1) using the telephone company service offering to call back a phone number until someone answers and a voicemail message is delivered, (2) sending an overnight mail package with a personalized invitation letter, and (3), making a home visit (where permission was granted on the original screening contact form) to each partner.

\section{DISCUSSION}

To date, no studies have focused on the recruitment of African American and Latino couples into an HIV/STI prevention intervention trial. This paper addresses this limitation by describing the preparation, development, and implementation of participant couple recruitment strategies comprising the protocol for Project Connect, a relationship-based HIV/STI prevention study. Preparation at the individual, community, study site, and research team levels allowed for careful consideration and reduction of barriers. The findings underscore the importance 
Table 3. Progressive Strategies for Difficult Recruitment Cases

Flexible scheduling: accommodate couples' schedules
with appointment times in the evening, on weekends,
and during drop-in periods for baseline interviews
Postscreening reminder phone calls: reluctant male
partners or couples who lost interest were reminded
of:
The altruism of their participation
The financial incentives provided
The potential benefits for their relationship
The confidential nature of study participation
Marketing approach: multiple reminder calls and mail-
ings were used to communicate continuing interest in
couple enrollment
Final special efforts:
Using pay-per-use phone company call back service
Sending an overnight mail package with a
personalized letter
Making a home visit

of eliciting input and feedback from couples in the target community in the developmental phase of the study to ensure not only the cultural relevance of recruitment strategies but also participant safety and confidentiality. There were no reported adverse events during the recruitment of couples for this study. In light of increasing literature documenting a relationship between IPV and HIV/STI risk behavior, ${ }^{38,39}$ the lack of adverse events suggests that couple recruitment without increasing the likelihood of IPV is possible. The findings also provide a description of some of the successful recruitment strategies and barriers experienced by couples entering an HIV prevention intervention. The ability to recruit and enroll 217 African American and Latino heterosexual couples into a multisession, relationshipbased HIV/STI prevention trial demonstrates the feasibility of engaging urban, minority couples into an RCT, despite concerns in the literature about barriers to engagement of minority men and women into RCTs. ${ }^{46,47}$

Lessons learned from this study have important implications for future couple recruitment efforts. First, as described here, successful recruitment protocols should be deliberately defined, articulated, and manualized, incorporating preparation phases at multiple levels (e.g., research team, study site, individual, and community) and specific strategies to be implemented. Second, couple recruitment with a protocol attuned to clinical issues, such as participant safety and confidentiality, is time and labor intensive.
We relied predominantly on a full-time team of one male and one female senior recruiter. In addition, intensive weekly supervision to monitor, review, and troubleshoot difficulties in the recruitment process with study investigators and consultants made immediate response to lagging recruitment deadlines and problem areas possible. Third, couple recruitment requires research staff to accommodate evening and weekend hours and to be more flexible and vigilant in terms of the follow-up required to reschedule missed appointments. Fourth, momentum in the recruitment process is critical. Maintaining communication and following up with eligible participants to complete an initial baseline interview within 2 weeks of screening seemed to be associated with more successful recruitment.

\section{Limitations}

A few study limitations are important to note. Although this paper describes the preparation, development, and implementation of couple recruitment strategies, it does not specifically test the relative success of each strategy by type of participant. This study was a first step toward a more rigorous recruitment methods analysis, which would make a valuable contribution to the field. Additionally, this study did not quantify sociodemographic characteristics of those participants who refused to be screened, thus limiting our knowledge of the representativeness of the enrolled study sample to the larger community from which the sample is drawn. We did compare aggregate data on the race and ethnicity of outpatient clinic population clientele (for the same years that study enrollment took place) with study enrollees, finding that fewer Latina women and more African American women were screened and enrolled into the study.

\section{CONCLUSIONS}

More research aimed at delineating and testing specific strategies for recruiting defined populations into clinical trials is needed. ${ }^{14}$ In future studies, we plan to implement quantitative measures to assess which strategies were more or less successful with different participant couples. Such future work will provide an empirical base for determining best practices for RCT recruitment. 
Implementing evidence-based strategies for study recruitment not only should strengthen recruitment and retention of vulnerable populations into RCTs but also will have the secondary benefits of enhancing the generalizability of research findings and improving overall cost-effectiveness of RCTs. Although this study adds to the literature on articulated recruitment strategies for couples, such strategies also need to be quantified and tested in more rigorous designs for their efficacy relative to alternative couple recruitment strategies (e.g., recruiting either male or female partners first, recruiting partners simultaneously). One unintended consequence of a recruitment protocol where the woman recruits her male partner, for example, is that the sample is likely biased toward male partners more willing to participate. When Krokoff ${ }^{31}$ implemented couple recruitment based on female recruitment of male partners into a marital therapy study, he found that compared with participating husbands, nonparticipating husbands were rated by their wives as less emotionally involved in their marriages. This supports the inference that successfully recruited male partners would be potentially more supportive of efforts to improve the health and safety of the relationship. We did not quantify the reasons for male partner nonparticipation, yet clearly a substantial number of women were unable to enroll because of their partners' objections. Thus, findings are not generalizable to all couples attending outpatient primary care clinics.

Finally, current software and database technology can be used to improve the science of RCT recruitment. Tracking databases allow for quantifying type, frequency, and duration of study participant contacts, as well as for case-based note taking to capture more qualitative detail on messages communicated to and from participants during the recruitment and enrollment process. Closer scrutiny to this level of data could provide a comparison of contact detail with successful enrollment by case, which could describe predictors for type, length, or frequency of contacts based on demographic indicators of participants.

\section{REFERENCES}

1. Allen S, Serufilira A, Bogaerts J, et al. Confidential HIV testing and condom promotion in Africa: Impact on HIV and gonorrhea rates. JAMA 1992;268:3338.
2. Deschamps M, Pape J, Haffner A, Hyppolite R, Johnson $\mathrm{W}$. Heterosexual activity in at risk couples for HIV infection. In: VII International Conference on AIDS, 1991; International AIDS Society, Florence, Italy, 1991: 318.

3. Ehrhardt AA, Exner T. Prevention of sexual risk behavior for HIV infection with women. AIDS 2000; 14:S53.

4. El-Bassel N, Witte SS, Gilbert L, et al. The efficacy of a relationship-based HIV/STD prevention program for heterosexual couples. Am J Public Health 2003;93:963.

5. El-Bassel N, Witte SS, Gilbert L, et al. HIV prevention for intimate couples: A relationship-based model. Families Systems Health 2001;19:379.

6. Harvey S. New kinds of data, new options for HIV prevention among women: A public health challenge. Health Educ 2000;27:539.

7. Higgins DL, Galavotti C, O'Reilly KR, et al. Evidence for the effects of HIV antibody counseling and testing on risk behaviors. JAMA 1991;266:2419.

8. Musaba E, Morrison CS, Sunkutu MR, Wong EL. Long-term use of the female condom among couples at high risk of human immunodeficiency virus infection in Zambia. Sex Transm Dis 1998;25:260.

9. Padian NS, O'Brien TR, Chang YC, Glass S, Francis D. Prevention of heterosexual transmission of human immunodeficiency virus through couple counseling. J Acquir Immune Defic Syndr 1993;6:1043.

10. Wingood GM, DiClemente RJ. Application of the theory of gender and power to examine HIV-related exposures, risk factors, and effective interventions for women. Health Educ Behav 2000;27:539.

11. Voluntary HIV-1 Counseling and Testing Efficacy Study Group. Efficacy of voluntary HIV-1 counseling and testing in individuals and couples in Kenya, Tanzania and Trinidad: A randomized trial. Lancet 2000;356:103.

12. Varmus H. NIH guidelines on the inclusion of women and minorities as subjects in clinical research. Fed Reg 1994;59:14508.

13. National Institutes of Health. NIH strategic research plan to reduce and ultimately eliminate health disparities. Washington, DC: Department of Health and Human Services, 2001.

14. Swanson MG, Ward AJ. Recruiting minorities into clinical trials: Toward a participant-friendly system. J Natl Cancer Inst Monogr 1995;87:1747.

15. Hatchett BF, Holmes K, Duran DA, Davis C. African Americans and research participation: The recruitment process. J Black Studies 2000;30:664.

16. Holder B, Turner-Musa J, Kimmel PL, et al. Engagement of African American families in research on chronic illness: A multisystem recruitment approach. Fam Process 1998;37:127.

17. Becker S. Couples and reproductive health: A review of couple studies. Stud Fam Plann 1996;27:291.

18. Thomas CR, Pinto HA, Roach M, Vaughn CB. Participation in clinical trials: Is it state of the art treatment for African Americans and other people of color? J Natl Med Assoc 1994;86:177. 
19. Thompson EE, Neighbors HW, Munday C, Jackson JS. Recruitment and retention of African American patients for clinical research: An exploration of response rates in an urban psychiatric hospital. J Consult Clin Psychol 1996;64:861.

20. Corbie-Smith G, Thomas SB, St. George DMM. Distrust, race, and research. Arch Intern Med 2002; 162:2458.

21. Gifford AL, Cunningham WE, Heslin KC, et al. Participation in research and access to experimental treatments by HIV-infected patients. N Engl J Med 2002;346:1373.

22. Sinclair S, Hayes-Reams P, Myers HF, Allen W, Hawes-Dawson J, Kington R. Recruiting African Americans for health studies: Lessons from the DrewRand Center on Health and Aging. J Ment Health Aging 2000;6:39.

23. Lovato LC, Hill K, Hertert S, Hunninghake DB, Probstfield JL. Recruitment for controlled clinical trials: Literature summary and annotated bibliography. Controlled Clin Trials 1997;18:328.

24. Levkoff SE, Levy BR, Weitzman PF. The matching model of recruitment. J Ment Health Aging 2000;6:29.

25. Miranda J, Azocar F, Organista KC, Munoz RF, Lieberman A. Recruiting and retaining low-income Latinos in psychotherapy research. J Consult Clin Psychol 1996;64:868.

26. Marin G, Marin BV. Research with Hispanic populations. Thousand Oaks, CA: Sage Publications, Inc, 1991.

27. Szapocznik J, Santisteban D, Rio A, Perez-Vidal A, Kurtines W, Hervis O. Bicultural effectiveness training (BET): An intervention modality for families experiencing intergenerational/intercultural conflict. Hispanic J Behav Sci 1986;8:303.

28. Sormanti M, Pereira L, El-Bassel N, Witte S, Gilbert L. The role of community consultants in designing an HIV prevention intervention. AIDS Educ Prev 2001; 13:311.

29. Preloran HM, Browner CH, Lieber E. Strategies for motivating Latino couples' participation in qualitative health research and their effects on sample construction. Am J Public Health 2001;91:1832.

30. Karney BR, Davila J, Cohan CL, Sullican KT. An empirical investigation of sampling strategies in marital research. J Marriage Fam 1995;57:909.

31. Krokoff LJ. The relationship of the husband's emotional involvement to participation on marital relationships. J Fam Issues 1990;11:182.

32. O'Farrell T, Cutter HS. Behavior and marital therapy for male alcoholics: Clinical procedures from a treatment outcome study in progress. Am J Fam Ther 1984;12:33.

33. DeVincenzi I. A longitudinal study of human immunodeficiency virus transmission by heterosexual partners. N Engl J Med 1994;331:341.

34. Kamenga M, Ryder RW, Jingu M, et al. Evidence of marked sexual behavior change associated with low
HIV-1 seroconversion in 149 married couples with discordant HIV-1 serostatus: Experience at an HIV counselling center in Zaire. AIDS 1991;5:61.

35. McMahon JM, Tortu S, Torres L, Pouget ER, Hamid R. Recruitment of heterosexual couples in public health research: A study protocol. BMC Med Res Methodol 2003;3:24.

36. Sobo EJ. Finance, romance, social support and condom use among impoverished innercity women. Hum Org 1995;54:115.

37. Kelly JA. Advances in HIV / AIDS education and prevention. Fam Rel 1995;44:345.

38. El-Bassel N, Gilbert L, Rajah V. The relationship between drug abuse and sexual performance among women on methadone: Heightening the risk of sexual intimate violence and HIV. Addict Behav 2003; 28:1385.

39. El-Bassel N, Gilbert L, Rajah V, Foleno A, Frye V. Fear and violence: Raising the HIV stakes. AIDS Educ Prev 2000;12:154.

40. NIMH Multisite HIV Prevention Trial. Quality control and quality assurance in HIV prevention research: Model from a multisite HIV prevention trial. AIDS 1997;11:s49.

41. Straus M, Hamby S, Boney-McCoy S, Sugarman D. The Revised Conflict Tactics Scales (CTS2): Development and preliminary psychometric data. J Fam Issues 1996;17:283.

42. Bograd M. Family system approaches to wife battering: A feminist critique. Am J Orthopsychiatry 1984;54:558.

43. Balcom DA, Healey D. The context of couples treatment for wife abuse. In: Mirkin MP, ed. The social and political contexts of family therapy. Boston: Allyn and Bacon, 1990, pg 121.

44. Herek GM, Capitanio JP, Widaman KF. HIV-related stigma and knowledge in the United States: Prevalence and trends, 1991-1999. Am J Public Health 2002;92:341.

45. NIMH Multisite HIV Prevention Trial. Reducing sexual risk behavior. Science 1998;280:1889.

46. Harris Y, Gorelick PB, Samuels P, Bempong I. Why African Americans may not be participating in clinical trials. J Natl Med Assoc 1996;88:630.

47. Shavers VL, Lynch CF, Burmeister LF. Factors that influence African-American willingness to participate in medical research studies. Cancer 2001;91(Suppl. 1):233.

Address reprint requests to: Susan S. Witte, Ph.D. Social Intervention Group Columbia University School of Social Work 1255 Amsterdam Aveue, Room 813 New York, NY 10027

E-mail: ssw12@columbia.edu 